\title{
Wavelet analysis and visualization of the formation and evolution of low total ozone events over northern Sweden
}

\author{
Beth L. Weinberg ${ }^{1}$, S. Roland Drayson ${ }^{1,2}$ and Katherine Freese ${ }^{3}$
}

\begin{abstract}
The formation and development of abnormally low total ozone events (LOEs), which typically last an average of 3-4 days, are analyzed to determine conditions under which these events may form. Wavelet analysis is performed on 13 years of daily total column ozone (TOZ) obtained by the Nimbus-7 Total Ozone Mapping Spectrometer each day of the record. With wavelet analysis, the contributions and relative phases of signal components may be ascertained at specific times of interest. Timescales relevant during LOEs are identified and the contributions and phase relations of all components present in the TOZ signal are compared for common patterns and features. For a location in Sweden, the formation of $96 \%$ of the 52 LOEs studied required that all components with timescales $(\tau)$ less than a maximum value $\left(\tau_{\max }\right)$ were present in their negative phases. This suggests that LOEs result from the simultaneous interaction of TOZdepleting processes. The number of interacting processes varies with season and location. In addition, two criteria are seen to prevail when LOEs form in this location. First, background conditions favorable for the development of an event exist; these occur when $\mathrm{TOZ}$ is in a spectral state where all components with $\Delta \tau_{1}<\tau<\tau_{\max }$ are in their negative phases. Second, a process takes place that can force the remaining short-timescale components $\left(\tau<\Delta \tau_{1}\right)$ into their negative phases. Processes associated with "initiating" timescales $\left(\Delta \tau_{1}\right)$ of $\approx 2-6$ days are found to be a key factor in the initiation of LOEs in this location. Although the background conditions are not uncommon, the likelihood that both criteria are simultaneously met is low and the occurrence of LOEs is indeed rare (averaging 4.7 events per year from 1980-1990).
\end{abstract}

\section{Introduction}

Short-lived, localized episodes of abnormally low and high values of total column ozone (TOZ) occur intermittently. High frequency fluctuations of $20-50 \%$ are not uncommon and localized events of extreme low TOZ, lasting from one to six days, are seen in certain locations (Rood, et al., 1992; Reck, et al., 1996). In this work, we answer some of the questions regarding the timescales of the forcing mechanism(s) of abnormally low total ozone events (LOEs) and the "conditions" under which LOEs form in different seasons and locations. LOEs are here considered to be spatially localized episodes of abnormally low TOZ compared to the average value $\langle\mathrm{TOZ}\rangle$ for the time period surrounding the event and will be further defined in the following sections.

Here, we present the results of a wavelet-based approach to identify, visualize and analyze the formation and development of LOEs. Traditional spectral methods determine the timescale content of a time series, but offer no information regarding the daily "activity" of each separate component. Since LOEs possibly re-

\footnotetext{
'Applied Physics Program, The University of Michigan, Ann Arbor.

'Department of Atmospheric, Oceanic and Space Sciences, The University of Michigan, Ann Arbor.

'Department of Physics, The University of Michigan, Ann Arbor.

Copyright 1996 by the American Geophysical Union.
}

Paper number 96GL02053

0094-8534/96/96GL-02053\$05.00 sult from the interaction of a number of processes with different timescales, the contributions and phase relations of all components present in the TOZ signal must be considered as a function of time in order to identify, or eliminate, specific processes involved in the formation of these events. The advantage of wavelet analysis over Fourier techniques is that the contributions and relative phases of low and high frequency components can be determined at specific times of interest, such as during LOEs. Thus, the spectral behavior of LOEs can be examined and the timescales associated with the development of LOEs determined. A number of locations around the globe are being studied. In this paper we present results for a location in northern Sweden (64.5N, 18E).

We apply wavelet analysis to spectrally decompose the 13-year time series of daily TOZ obtained by the Nimbus-7 Total Ozone Mapping Spectrometer (TOMS) each day of the record. The database used is GRIDTOMS Version 6 which contains average daily $\mathrm{TOZ}$ values in Dobson Units (DU) for grid cells measuring $1^{\circ}$ latitude by $1.25^{\circ}$ longitude (McPeters, et al., 1993; Herman, et al., 1991). The uncertainty associated with each daily grid value is not more than $\pm 5 \%$. The percentage of missing data is $\approx 3.8 \%$ over the 11-year interval (Jan. 1980-Dec. 1991) in which LOEs have been examined in this work. We have linearly interpolated the series to account for missing values; LOEs that occurred near these data values are noted.

\section{The wavelet method}

The mathematical foundations of wavelet analysis can be found in a number of references including Daubechies (1988) and Kaiser (1995). Wavelet analysis is starting to be used extensively in the study of geophysical signals which are known to be non-stationary and exhibit features with multiple timescales (Lau and Weng, 1995; Gao and Li, 1993; Bolton, et al., 1995; Kumar, 1995, Lou and Rial, 1995).

The wavelet method expands a time series in terms of basis functions, $\psi\left(t^{\prime}\right)$, that are well-localized in both time and frequency and have zero mean: $\int \psi\left(t^{\prime}\right) d t^{\prime}=0\left(-\infty \leq t^{\prime} \leq \infty\right)$. Components of the signal are defined with respect to the particular wavelet basis function used. In this work, we use the "Mexican Hat" function: $\psi\left(t^{\prime}\right)=\left[1-16\left(t^{\prime}\right)^{2}\right] \exp \left[-8\left(t^{\prime}\right)^{2}\right]$. On day $n$, the wavelet transform coefficient of the component with timescale $\tau$ is defined as the convolution integral of the time series being analyzed, $f(t)$, with the complex conjugate $\left(\psi^{*}\right)$ of the wavelet basis function: $W(\tau, n)=|\tau|^{-}$ ${ }^{1 / 2} \int \psi^{*}[(\mathrm{t}-\mathrm{n}) / \tau] \mathrm{f}(\mathrm{t}) \mathrm{dt}$, where $\mathrm{t}^{\prime}=[(\mathrm{t}-\mathrm{n}) / \tau]$ (here, in days). The parameters $\tau$ and $n$ are used to adjust the timescale (resolution) and location of the wavelet. By adjusting $\tau$, the shape of the wavelet envelope is compressed or stretched allowing fluctuations with different timescales to be resolved from the signal. The full-width of this wavelet's central peak is $\tau / 2$. The energy normalization factor $|\tau|^{-1 / 2}$ keeps the energy of the scaled wavelets the same as the original. The resulting wavelet transform contains information regarding component amplitude and phase (relative to the analyzing wavelet) as a function of both time and scale.

Briefly, the results of a wavelet analysis of geophysical data should not depend on the choice of basis function (see Lau and 


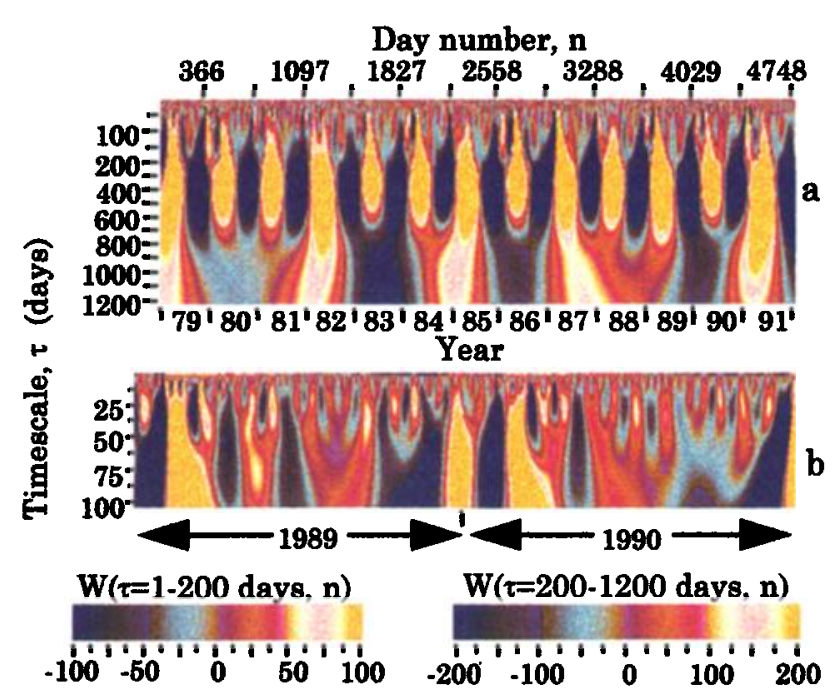

Figure 1. Wavelet transform patterns for location in Sweden. a) Transforms representing 13 years of daily $W(\tau, n)$ for $1<\tau \leq 1200, \tau$ is timescale and $n$ is the specific day number in the time series, $1 \leq n \leq 4748$ days. Note difference in the interval along the timescale axis for $1 \leq \tau \leq 200$ days and $200<\tau \leq 1200$. b) Magnified small to medium-scale patterns for the years 1989.

Weng, 1995 for more details). However, properties of the basis function need to be taken into account to correctly interpret the resulting wavelet transform and certain functions are better-suited for obtaining specific results. For example, symmetric functions (e.g. Mexican Hat) are useful in identifying regions of maximum curvature (Hagelberg and Gamage, 1994). The functional properties of the Mexican Hat wavelet and the features of time localization and adjustable scale, inherent to wavelet analysis, are extremely useful in the study of LOEs as they allow the identification of timescales relevant during the event.

\section{The wavelet transform of TOMS data}

Wavelet transform patterns for the location in Sweden are shown in Figure 1. In these patterns, the wavelet coefficient is plotted as a function of timescale along the vertical axis and time on the horizontal. (To aid the visualization of small scale structures, the range of the wavelet coefficients' magnitude represented by the color bars is different for $1 \leq \tau \leq 200$ and $200<\tau \leq 1200$ days

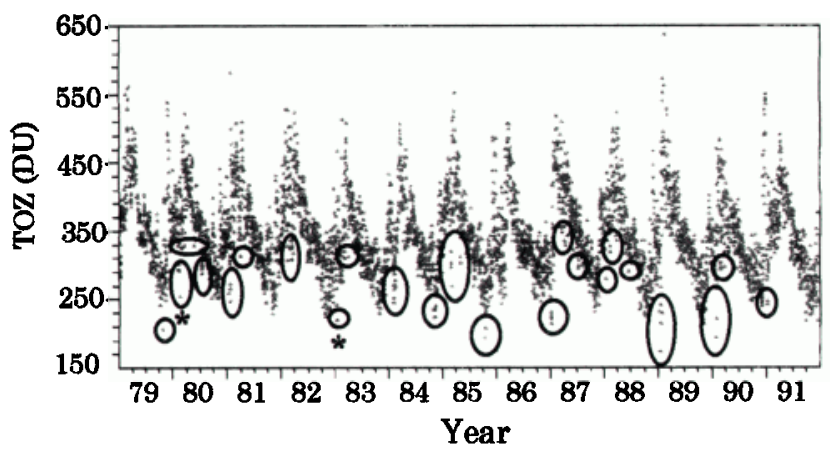

Figure 2. a) The 13-year time series of daily TOZ. The uncertainty associated with each grid cell value is not more than $\approx 5 \%$. For clarity, error bars have not been added. A number of LOEs are indicated-some ovals contain more than one event (asterisks denote events that occurred near missing data values).

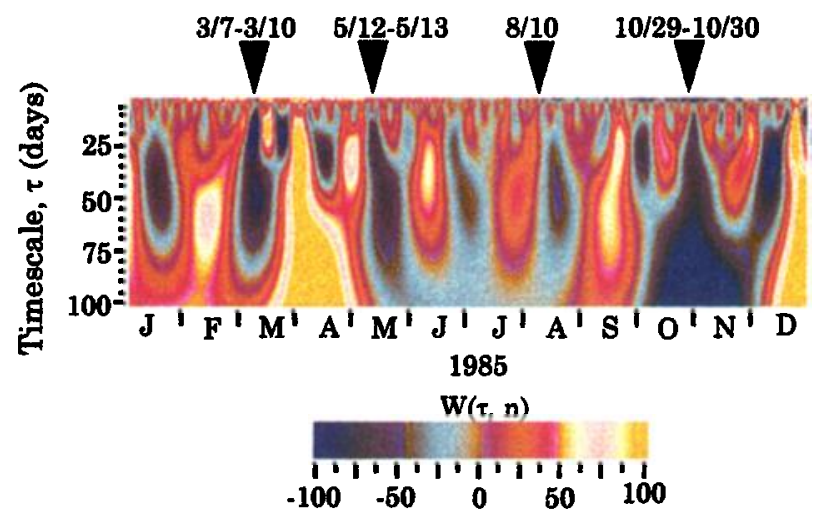

Figure 3. Wavelet transform pattern for 1985. Arrows highlight four LOEs.

in Fig. $1 \mathrm{a}$ and $1 \mathrm{~b}$.) The corresponding 13-year time series of daily TOZ is shown in Figure 2. As can be seen, a large amount of information is contained in the wavelet patterns alone. Features on all timescales examined in this work ( $1 \leq \tau \leq 1200$ days) occur in the TOZ signal and are present in the wavelet patterns with timevarying contributions. The amplitude of $W(\tau, n)$ varies with $\tau$ and season, and thus indicates when fluctuations with corresponding timescales are most/least active.

Distinguishable from the background of the wavelet patterns are regions where the distribution of wavelet coefficients assemble into oval-shaped structures that occur intermittently or systematically as functions of time. $\tau$ values near the center of these structures, $\tau_{c}$, (which have the strongest wavelet amplitudes) correspond to timescales of fluctuations in the TOZ signal. For example, the most obvious structure, centered about a timescale of $\approx 370$ days, corresponds to the annual oscillation of TOZ and occurs regularly throughout the wavelet pattern and time series. A few cycles of the quasi-biennial oscillation (QBO) in TOZ are discernible as structures with $\tau_{\mathrm{c}} \approx 1000$ days. Structures affiliated with the annual oscillation and the QBO merge during years when these two processes are in phase. Another frequently occurring structure is that which corresponds to timescales centered about 25-32 days. Chandra (1991) has shown that TOMS derived TOZ has a "measurable response to solar UV forcing on a timescale of a solar rotation (27 days)." It is, therefore, reasonable to assume that solar rotation is one contributor to components associated with these structures. Processes that contribute to the TOZ signal during localized time intervals appear as intermittent, or systematically occurring, structures in the wavelet patterns of Figures 1 and 3 . For example, structures with $\tau_{c} \approx 2-6,5-15,25-32,45-60$ and $70-75$ days are readily observed.

\section{Wavelet analysis of LOEs}

The focus of this work is on the formation and development of abnormally low total ozone events. The magnitude of TOZ which constitutes an abnormally low value depends on season and location and is here defined to fall $\sim 2.0 \sigma$ (or more) below $<\mathrm{TOZ}\rangle$, where $\sigma$ is the standard deviation of the difference between the actual TOZ value and the average background value on day $n$ ( $\sigma$ is smallest during the months of June through September and largest during mid-February through mid-May). We are presently concerned with the formation of LOEs that are temporally isolated, i.e. the uncertainty associated with each low value in the event may not significantly overlap with nearby $\mathrm{TOZ}$ values outside of the 
event (non-isolated events have not been included in this study). Events may be either a single, temporally isolated $\mathrm{TOZ}$ value of abnormally low magnitude, or a temporally isolated group of $\mathrm{TOZ}$ values that contains days of abnormally low TOZ.

Intermittent, isolated events of abnormally low and high TOZ can be seen in the 13-year time series for the site in Sweden. Some of the LOEs that occurred in this location are indicated in Figure 2. We have analyzed the spectral evolution of 52 events between January 1980 and December 1990 . The average number of events per year over this time period is 4.7 . $28.8 \%$ of the events occurred between early October and early February, $46.1 \%$ between mid-February and May, and $25 \%$ between June and early October. That the highest percentage of events occurred during the "spring" is not surprising since this is when the daily variability in Sweden's TOZ is greatest. The average lifetime of the events was 3.4 days and ranged from 1-7 days. During the events, negative deviations of $40 \mathrm{DU}$ to $163 \mathrm{DU}$ below $\angle \mathrm{TOZ}>$ were measured. The greatest negative deviations occurred between January and May.

Wavelet patterns allow the identification and visualization of the LOEs' development. The relative contributions and phase relations of all components of the TOZ signal with timescales up to 200 days are analyzed on days of, and surrounding, the events for common patterns and features which define the "spectral conditions" under which LOEs are most likely to form. The 1985 wavelet pattern in Figure 3 highlights four typical LOEs. The component contributions and phase relations present in the time series during the interval surrounding the events can be seen in these patterns and also in the corresponding plots of daily $W(\tau, n)$ shown in Figure 4. Plots of $W(\tau, n)$ detail the evolution of LOEs from a spectral standpoint.

LOEs appear to form when there is a simultaneous interaction of a number of negative-phase fluctuations. This is indicated by the common spectral patterns/features exhibited by the LOEs studied: all components with timescales less than some maximum value, $\tau_{\max }$ (which varies with season and location as does the number of interacting fluctuations) are simultaneously present with negative wavelet coefficients. For the location in Sweden, $\tau_{\max }$ is typically greater than 150 days from September through February, with the exception of December and January where the average $\tau_{\max } \approx 47$ days. During spring and summer $\tau_{\max }$ ranges from $\approx 25$ days to over 100 days.

LOEs seem to be preceded by the occurrence of fluctuations with initiating timescales $\left(\Delta \tau_{\mathrm{i}}\right) \approx 2-6$ days. Components with $\Delta \tau$ $\approx 2-4$ days are connected with the development of all LOEs in this location and commonly are the initiators, suggesting that processes associated with these timescales are key to the formation of LOEs. Events with lifetimes $\approx \Delta \tau_{1}$ appear to dissipate when the phases of the initiating components change from negative to positive or their contributions become negligible. Events of longer duration are deepened and/or sustained by components with $\tau>\Delta \tau_{1}$ acting slightly out-of-phase with the initiating components (i.e., sequentially occur in their respective minima after the initiation of the event). During sustained LOEs, the phase of components with timescales much less than the lifetime of the event may change signs a number of times. These events also appear to dissipate when the initiating components enter their positive phases, but only after the sustaining components have passed through their respective minima and their contributions are no longer sufficient to sustain the LOE.

The temporal behavior exhibited by LOEs is detailed in the formation sequences of Figure 4. For example, on the days preceding the event of March 7-10, 1985, components with $5<\tau<80$ days are in their negative phases (components with $4 \leq \tau \leq 9$ days are not yet active, i.e. have negligible contribution to the signal). These conditions are "prime" for the formation of an LOE, which is now dependent on the simultaneous occurrence of a TOZdepleting process that can force components with $\tau \leq 5$ days into their negative phases. This happens on March 7 when negative values of all wavelet coefficients with $\tau<\tau_{\max }$ are observed (although the components with $2 \leq \tau \leq 5$ days still have negligible contributions. By March 9, $\tau \approx 4-15$ days contribute strongly to the signal. These components may possibly induce the process associated with $\Delta \tau_{1}=2-4$ days which appears on March 10 with a large negative contribution and drives TOZ to only $64 \%$ of $\langle\mathrm{TOZ}\rangle-a$ negative deviation of 145DU (for comparison, the annual fluctuation of TOZ is roughly $\pm 120 \mathrm{DU}$ in this location). The event dissipates on March 11 when components with $\Delta \tau=2-4$ days enter their positive phases. Processes associated with structures centered on timescales of $\approx 2-4,12,15-20$ and 45-60 days are present in the time interval surrounding this event (Figure 3 ). During the event, structures with $\tau_{\mathrm{o}} \approx 2-4,15-20$ and 45-60 days merge indicating they are in approximate phase. The second event detailed in Figure 4 occurred October 28-30, 1985 and follows a similar formation pattern.

The three wintertime events of 1989 and 1990 are examples of sustained/deepened LOEs and have been attributed to the interacting feedback processes of forced planetary wave activity and the ozone-depleting effects of polar stratospheric clouds (PSCs) (Rood, et al., 1992 and references therein). These events exhibit
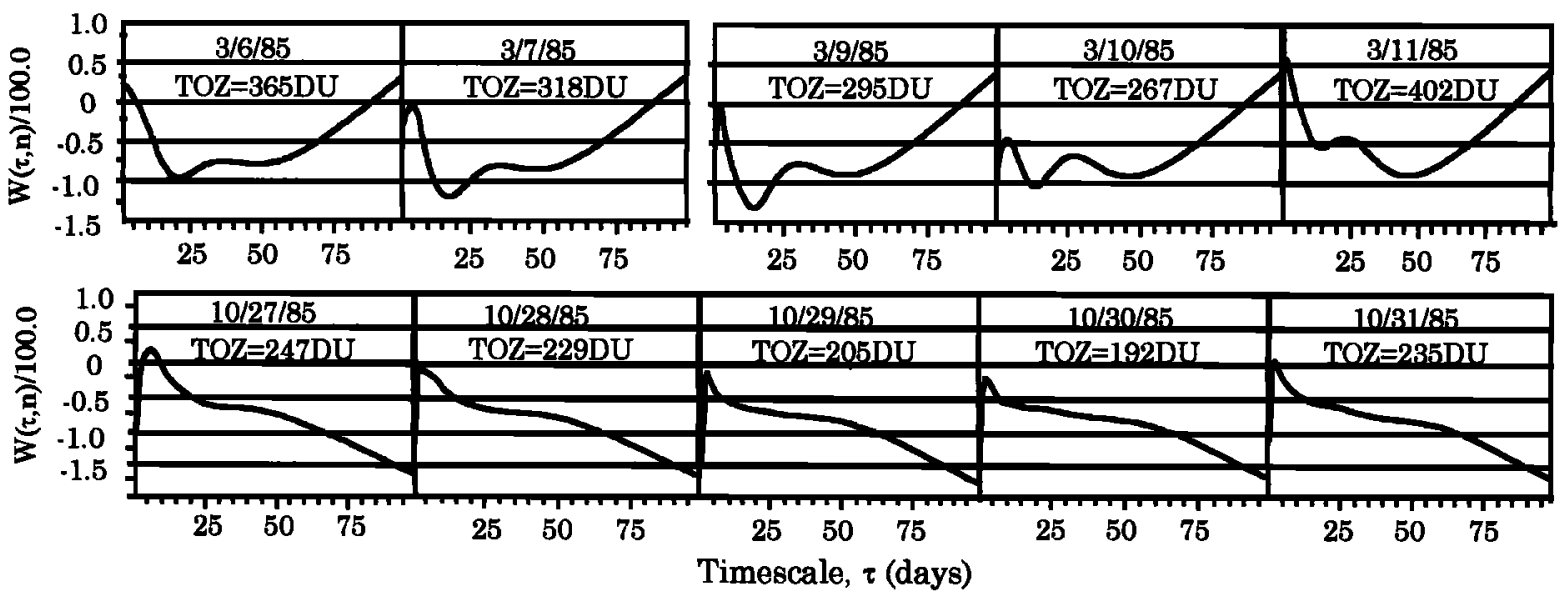

Figure 4. Formation sequences for two 1985 events. 
depletions well over $100 \mathrm{DU}$ with measured $\mathrm{TOZ}$ values less than $60 \%$ of $\langle\mathrm{TOZ}>$. Typical wavelet coefficients, $\mathrm{W}(\tau, \mathrm{n})$, for components with $\tau=\Delta \tau_{1}$ are between -20.0 and -30.0 during winter/earlyspringtime LOEs in this location. During the above mentioned events of 1989 and $1990, W\left(\Delta \tau_{1}, n\right)$ was approximately $-50.0-a$ $40 \%$ to $60 \%$ difference from typical wintertime values. Coefficients with these negative magnitudes are not seen during seasons in which PSCs are not present. It, therefore, may be possible to determine if PSCs were present, or induced, during events which exhibit large negative values of $W\left(\Delta \tau_{1}, n\right)$ such as the event of March 1985 detailed in Figure 4. Quantitative analyses of locations where PSC formation has been confirmed need to be performed utilizing appropriate wavelet basis functions in order to determine if the presence of PSCs can be inferred from wavelet coefficients.

\section{Discussion}

We have presented initial results of a wavelet-based approach to identify, visualize and analyze the formation and development of abnormally low total ozone events. Timescales of fluctuations relevant during the lifetime of LOEs have been ascertained and the spectral conditions under which LOEs are most likely to form in different seasons determined. This information will be useful in the attempt to infer which physical mechanisms contribute (do not contribute) to the occurrence of these events. In principle, the timescales of the fluctuations in TOZ may differ from the timescales of the physical mechanisms that forced them. In future work, it may be possible to obtain more concrete information regarding the forcing mechanisms by comparing the results of wavelet scale-analyses of $\mathrm{TOZ}$ and of other data sets known to influence ozone (e.g. temperature profiles, solar irradiance, changes in tropopause height, etc.).

Winter and early springtime events in northern Sweden are commonly attributed to the interaction of planetary waves due to anticyclonic disturbances and the ozone-depleting effects of PSCs. PSCs are known to introduce errors in TOMS data which may lead to derived TOZ values lower than actual by $\approx 2-6 \%$ depending on the composition of the PSC (type-I or II) (McPeters, et al., 1993). As indicated by the similarity of wavelet patterns in seasons and locations without PSC formation, and comparison with previous studies of the events of January-February 1989 and February 1990 , the presence of PSCs does not alter the overall features and spectral conditions common to the formation of the LOEs studied: 1) LOEs are initiated by processes associated with small timescale components of the TOZ signal, 2) on the day of formation of an LOE all components with timescales less than $\tau_{\max }$ are in their negative phases, which physically suggests that 3 ) LOEs result from the simultaneous interaction of TOZ-depleting processes versus being the result of a single process.

Specifically, for the location studied in Sweden, we find that $96 \%$ of the LOEs studied were forced when initiating TOZdepleting processes represented by timescale components of $\approx 2-6$ days occurred exactly when background spectral conditions were such that all components with timescales between the initiating values, $\Delta \tau_{1}$, and a maximum value, $\tau_{\max }$ (which varies with season) were also present in their negative phases. These background conditions may be pre-existing or occur simultaneously with the initiating process. These prime conditions are not unique to time intervals surrounding LOEs - they are also noted during times of average and low TOZ. Events of abnormally low total ozone occurred following days of average TOZ as well as days with lower than average $\mathrm{TOZ}$ values. This suggests that $\mathrm{TOZ}$ is not required to be below a local threshold value prior to the formation of an LOE. Although conditions favorable to the formation of these events are not uncommon, the likelihood that a small-scale TOZdepleting process will simultaneously occur with sufficient magnitude is low, as indicated by the small average number of events (4.7) per year from January 1980 through December 1990.

Acknowledgments. We thank R. McPeters, W. Kubn, D. Jenkins, R. Clarke, W. Gao and R. Reck for their helpful comments and discussions regarding this manuscnpt, data and analysis; and The Ozone Processing Team for supplying the TOMS data. In addition we thank $M$. Ko and the reviewers of this manuscript for their time, suggestions and comments. This work was supported by the U.S. Department of Energy's Fellowships for Global Change Program administered by Oak Ridge National Laboratory. Initial phases of this work were supported in part by the Atmospheric Chemistry Program of the U.S. Department of Energy.

\section{References}

Bolton, E.W., K.A. Maasch and J.M. Lilly, A wavelet analysis of pilopleistocene climate indicators: A new view of periodicity evolution, Geophys. Res. Lett., 22(20), 2753-2756, 1995.

Chandra, S., The solar uv related changes in total ozone from a solar rotaton to a solar cycle, Geophys. Res. Lett., 18(5), 837-840, 1991.

Daubechies, I., Ten Lectures on Wavelets, SIAM, Philadelphia, 357 pp., 1992.

Gao, W. and B.L. Li, Wavelet analysis of coherent structure at the atmosphere-forest interface, J. Appl. Meteorol., 32(11), 1717-1725, 1993.

Hagelberg, C.R. and N.K.K. Gamage, Applications of structure preserving wavelet decompositions to intermittent turbulance: A case study, in Wovelets in Geophysics, Eds. E. Foufoula-Georgiou and P. Kumar, pp. 45-80, Academic Press, Mass., 1994.

Herman, J.R., R. Hudson, R McPeters and R. Stolarski, A new selfcalibration method applied to TOMS and SBUV backscattered ultraviolet data to determine long-term global ozone changes, $J$. Geophys. Res., 96(D4), 7531-7545, 1991.

Kaiser, G., A Friendly Guide to Wovelets, Birkhauser, Boston, 300 pp, 1994.

Kumar, P., A wavelet based methodology for scale-space anisotropic analysis, Geophys. Res. Lett., 22(20), 2777-2780, 1995.

Lau, K.M. and H. Weng, Climate signal detection using wavelet transform: How to make a time series sing, Bulletin Am. Meteorol. Soc., 76(12), 2391-2402, 1995.

M. Lou and J.A. Rial, Application of the wavelet transform in detecting multiple events of microearthquake seismograms, Geophys. Res. Lett., 22(16), 2199-2202, 1995.

McPeters, R.D., A.J. Krueger, P.K. Bhartia, J.R. Herman, A. Oaks, Z. Ahmad, R.P. Cebula, B.M. Schlesinger, T. Swissler, S.L. Taylor, O. Torres and C.G. Wellemeyer, The Nimbus-7 Total Ozone Mapping Spectrometer (TOMS) Data Products User's Guide, NASA ref. publ. $1323,1993$.

Reck R.A., B.L. Weinberg, R.M. Bornick, G. Wen and J.E. Frederick, A new approach to the characterization of long-term changes in total atmospheric ozone: Determination and application of frequency distribution, Atmos. Environment, in press, 1996.

Rood, R.B., J.E. Nielsen, R.S. Stolarski, A.R. Douglass, J.A. Kaye and D.J. Allen, Episodic total ozone minima and associated effects on heterogeneous chemistry and lower stratospheric transport, $J$. Geophys. Res., 97(D8), 7979-7996, 1992.

S.R. Drayson, Department of Atmospheric, Oceanic and Space Sciences, The University of Michigan, Ann Arbor, MI 48109-2143. (email: drayson@engin.umich.edu)

K. Freese, Department of Physics, The University of Michigan, Ann Arbor, MI 48109-1120. (e-mail: ktfreese@umich.edu)

B.L. Weinberg, Applied Physics Program, The University of Michigan, Ann Arbor, MI 48109-1120. (e-mail: weinberg@engin. umich.edu)

(Received April 11, 1996; revised June 11, 1996;

accepted June 17, 1996.) 